\title{
Preface for the Special Issue on Games and AI
}

\author{
Mark H.M. Winands ${ }^{\mathrm{a}}$, Yngvi Björnsson ${ }^{\mathrm{b}}$, Karl Tuyls ${ }^{\mathrm{a}}$ \\ ${ }^{a}$ Department of Knowledge Engineering, Faculty of Humanities and Sciences, Maastricht University, Maastricht, \\ The Netherlands \\ ${ }^{b}$ School of Computer Science, Reykjavik University, Reykjavik
}

Ever since humans achieved some degree of civilization, they have played games. The two most important reasons for games to be played are their intellectual challenge and their entertainment value. For the first reason games are used as a testing ground for computational intelligence. Since the 1950s the AI community compares the computer performance with the human performance (Schaeffer, 2001), or otherwise stated: since the birth of AI computational intelligence is measured with respect to human intelligence. Shannon (1950) and Turing (1953) were the first to describe a chess-playing program, while Samuel (1959) wrote the first game-playing program in the domain of Checkers. In the beginning most AI research in games concentrated on abstract games like Chess and Checkers. Later on (in the 1970s) Backgammon and Bridge were added to this list, in particular since they possessed additional features, viz. non-determinism and imperfect information, respectively. These kind of games offer a pure abstract competition, with an exact closed domain (i.e., well-defined rules). The game state is easy to represent and the possible actions are known.

In the last years we observed two shifts in the domain of Games and AI. First, there is the research shift from trying to outplay the (human) opponent to entertaining the human player. Second, a shift was seen from the classic abstract games towards video games. Role-playing, real-

Email addresses:

m.winands@maastrichtuniversity.nl (Mark H.M. Winands), yngvi@ru.is (Yngvi Björnsson), k.tuyls@maastrichtuniversity.nl (Karl Tuyls) time strategy, and sport games have become increasingly more popular as a test domain for AI research. This is partly so because these games offer new challenges, such as real-time pathfinding and adversarial real-time planning. But it also stems from the fact that this subdomain has more resources (i.e., these games constitute a multibillion enterprise) to do research. Whatever the case, all games will remain an intriguing subject for AI research in the future.

The five articles for this special issue deal with these wide range of aspects in Games and AI. The first two papers discuss improving computational intelligence to outperform an intelligent opponent. The first one tackles a deterministic board game, while the second one deals with the abstract but non-deterministic game of Billiards. The last three papers focus on improving the entertainment aspect of a game in relationship with AI. We discuss them in detail below.

In the first article of this special issue, $K u$ nihito Hokia and Masakazu Muramatsu discuss the efficiency of three forward-pruning techniques, i.e., futility pruning, null-move pruning, and latemove reduction. They analyze these techniques in the game of Shogi, a Japanese chess variant. They show that these techniques combined with $\alpha \beta$ search reduce the effective branching factor of Shogi endgames to 2.8 without sacrificing much accuracy of the search results. When comparing the performance in Shogi with the performance in Chess, the authors conclude that the the forwardpruning techniques reduce the effective branching factor more effectively in Shogi than in Chess.

The next article, by Jean-François Landry, Jean- 
Pierre Dussault, and Philippe Mahey tackles the game of Billiards. This game has offered many interesting challenges to both communities of AI and optimization. The authors propose in their paper a refined controller for Billiards based on robust optimization combined with specific adjustments in order to take advantage of the domain knowledge. A multi-objective formulation of a robust controller is presented to provide the tools needed to execute any desired shot on the table, as part of a two-layered approach for the game of Billiards.

A survey of player behavioral modeling is given in the article written by Sander C.J. Bakkes, Pieter H.M. Spronck, and Giel van Lankveld. This type of modeling has grown from a means to improve the playing strength of computer programs that play classic games (e.g., Chess), to a means for impacting the player experience and satisfaction in video games, as well as in cross-domain applications such as interactive storytelling. In this context, player behavioral modeling is concerned with two goals, namely (1) providing an interesting or effective game AI on the basis of player models, and (2) creating a basis for game developers to personalize gameplay as a whole, and creating new user-driven game mechanics. In their article, the authors provide an overview of player behavioral modeling for video games by detailing four distinct approaches, i.e., (1) modeling player actions, (2) modeling player tactics, (3) modeling player strategies, and (4) player profiling. The authors conclude the article with an analysis on the applicability of the approaches for the domain of video games.

Li Zhang and Alamgir Hossain argue that sensing social representations (e.g., relationships and common interests) of a conversation and recognizing affect and metaphor from context are challenging but essential for the building of an intelligent agent with emotion and social intelligence. In the article, the authors report contextual affect detection based on emotion modeling of personal and social improvisational context. The authors also discuss the cooking and sensory metaphor (including temperature, light, taste, and smell metaphors) interpretation. The overall affect sensing model has been embedded in an AI agent interacting with human users. The evaluation results indicated that the new development made the AI agent perform generally better in improvisational interaction.

Finally, Hiroyuki Iida, Takeo Nakagawa and Kristian Spoerer propose in their article two different kinds of novel information dynamic models, for two team (or player) games, based on fluid mechanics. They analyze the five Base Ball games in the World Series 2010 using the models, finding that the first model represents one game group where information of the game outcome increases quite rapidly with increasing the game length near the end and takes the full value at the end. The second model represents another game group where information gradually approaches to the full value at the end. Three game-progress patterns are identified according to information pattern in the five Base Ball games, called "balanced", "seesaw" and "one-sided" games. In a "balanced game", neither team scores during the game. In a "seesaw game", one team leads, then the other team leads, and this may be alternated. In a "one-sided game", only one team scores. It is suggested that the present models make it possible to discuss the information dynamics in games, and also problems starting from zero information and ending with full information.

\section{References}

Samuel, A., 1959. Some studies in machine learning using the game of checkers. IBM Journal of Research and Development 3, 210-229.

Schaeffer, J., 2001. A gamut of games. AI Magazine 22, 29-46.

Shannon, C., 1950. Programming a computer for playing chess. Philosophical Magazine 41, 256-275.

Turing, A., 1953. Digital computers applied to games, in: Bowden, B. (Ed.), Faster Than Thought, Pitman Publishing, London, England. pp. 286-297. 